\title{
Automated Drawing of Railway Schematics using Numerical Optimization in $\mathrm{SAT}^{\star}$
}

\author{
Bjørnar Luteberget ${ }^{1}$, Koen Claessen ${ }^{2}$, and Christian Johansen ${ }^{3}$ \\ 1 Railcomplete AS \\ ${ }^{2}$ Chalmers University of Technology, Gothenburg, Sweden \\ 3 University of Oslo, Norway \\ bjlut@railcomplete.no, koen@chalmers.se, cristi@ifi.uio.no
}

\begin{abstract}
Schematic drawings showing railway tracks and equipment are commonly used to visualize railway operations and to communicate system specifications and construction blueprints. Recent advances in on-line collaboration and modeling tools have raised the expectations for quickly making changes to models, resulting in frequent changes to layouts, text, and/or symbols in schematic drawings. Automating the creation of high-quality schematic views from geographical and topological models can help engineers produce and update drawings efficiently. This paper describes three methods for automatically producing schematic railway drawings with increasing level of quality and control over the result. The final method, implemented in the tool that we present, can use any combination of the following optimization criteria, which have different priorities in different use cases: width and height of the drawing, the diagonal line lengths, and the number of bends. We show how to encode schematic railway drawings as an optimization problem over Boolean and numerical domains, using combinations of unary number encoding, lazy difference constraints, and numerical optimization into an incremental SAT formulation.

We compare resulting drawings from each of the three approaches, applied to models of real-world engineering projects and existing infrastructure. We also show how to add symbols and labels to the track plan, which is important for the usefulness of the final outputs. Since the proposed tool is customizable and efficiently produces high-quality drawings from railML 2.x models, it can be used (as it is or extended) both as an integrated module in an industrial design tool like RailCOMPLETE, or by researchers for visualization purposes.
\end{abstract}

\section{Introduction}

Engineering schematics of railway track layouts are used for several purposes: serving as construction blueprints, visualizations on train dispatch workstations, infrastructure models in timetabling software, specifications for interlocking control systems, and more. Because of the large distances involved, geographically

\footnotetext{
* The first author was partially supported by the project RailCons - Automated Methods and Tools for Ensuring Consistency of Railway Designs, with number 248714 funded by the Norwegian Research Council and Railcomplete AS.
} 
accurate drawings are not always suitable for communicating an overview that can help with analyzing and reasoning about the railway models. Instead, many disciplines use schematic representations of infrastructures to provide a compressed overview, e.g., shortening sections of the railway that have low information density. Fig. 1 compares a geographically correct drawing against two alternative schematic renderings (for two purposes) of the same model. Producing schematic drawings like these involves practical and aesthetic trade-offs between intended structure, simplicity, and geographical accuracy.

Perhaps the most well-known railway schematics are the metro maps for passengers, popularized by the iconic Tube Map of the London Underground. When designing metro maps, removing and compressing geographical information better conveys topological structure (e.g., useful for finding transfers) and sequential information along lines (e.g., for finding your stop).

Methods for automatically producing metro maps have been surveyed in [23]. The main approaches are iterative and force-directed algorithms for gradually transforming a geographical network map into a simpler presentation $[2,7]$, and mixed integer programming methods for finding exactly grid-structured and rigidly optimized solutions $[14,16]$. For railway drawings the convention is to use only horizontal, vertical, and diagonal lines (at $45^{\circ}$ ). The problem of drawing graphs optimized for size and/or bends using only horizontal and vertical lines (so-called orthogonal drawings) can be solved by efficient algorithms [21], but adding diagonal lines in general makes the problem NP-complete [14,15].

Schematic railway drawings used for engineering are usually more strictly constrained than metro maps, but still have large variety in different versions produced for different engineering use cases, project stages, and operational scenarios. Especially in construction projects for new railway lines or upgrades,
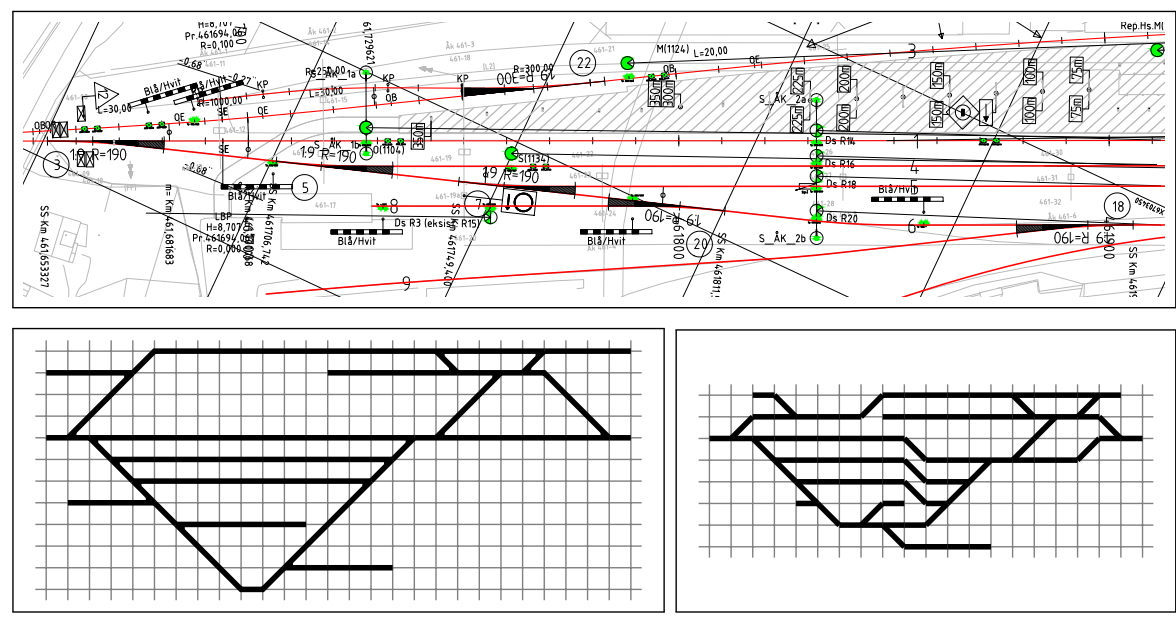

Fig. 1. Example cut-out from a geographical railway drawing (top) and two corresponding full-station schematic layouts, optimized for bends (bottom left) and optimized for height/width (bottom right). See on page 15 our tool's optimization options. 
frequent changes are made in coordinated 2D, 3D, geographical, and schematic models of the railway infrastructure. This can cause much repeated manual work in updating and cross-checking these models after every change in the construction design work in several engineering and construction categories, such as tracks, signaling and interlocking, catenary, cables, telephony.

Automatically producing consistent and high-quality schematics from other models has great potential to increase the efficiency and quality of the documentation, speed up cross-discipline communication during design and construction phases, and also opens up for easier data transfer to other tools. For example, an engineer working on a geographical CAD model may be hindered in performing capacity analysis because importing a network model into a capacity tool may require also inputting a track plan for simulation overview (see, e.g., [12]).

In this paper we develop methods for producing a type of schematic track plan which is suitable for infrastructure within a single corridor, meaning that each point on each track can be located on a common linear axis. We call this a linear schematic drawing (see Definition 1). This is a common drawing used for many purposes in construction projects, where drawings typically show placement of tracks and track-side equipment on a single station or along a single corridor. More generally, this problem concerns network structures that are oriented along a linear axis, such as highways, railways, or public transit systems, but may also be extended to encompass routing in electronic design (see e.g. the problem description for VLSI routing in [17]). On larger scales with multiple corridors, the visualization may be split into individual corridors, as in our setting, but for some applications, such as an overview of a national railway network or a city metro network, the single corridor assumption will not work well, and other approaches (see e.g. [14,16]) may be more relevant.

Linear schematic drawings specifically have little coverage in the literature. A specialized algorithm presented in [6] computes corridor-style drawings, but does not guarantee that switch shapes are preserved, and does not offer choice in optimization criteria. For comparison, we apply our method to examples taken from [6] (see Fig. 11). Another algorithmic approach described in [20] has similar goals, but does not automatically produce high-quality results and relies on interactive guidance from the user and manual post-processing.

Graph drawing techniques (see $[8,9]$ for a general overview) have been developed for a great number of different use cases. Most closely related to engineering drawings are the orthogonal layout methods (see e.g. $[18,21]$ ) and storyline visualizations (see e.g. [22]). However, most approaches from the graph drawing literature, including orthogonal layout methods, produce outputs that have a distinct style and are not suited to be customized to adhere to engineering drawing conventions.

Instead, we have solved the problem by modeling engineering drawings as mathematical optimization problems using constraints formulated as Boolean satisfiability and difference constraints. We present how different available constraint programming systems can be used to express our constraints, solve optimization problems, and produce high-quality engineering drawings. 


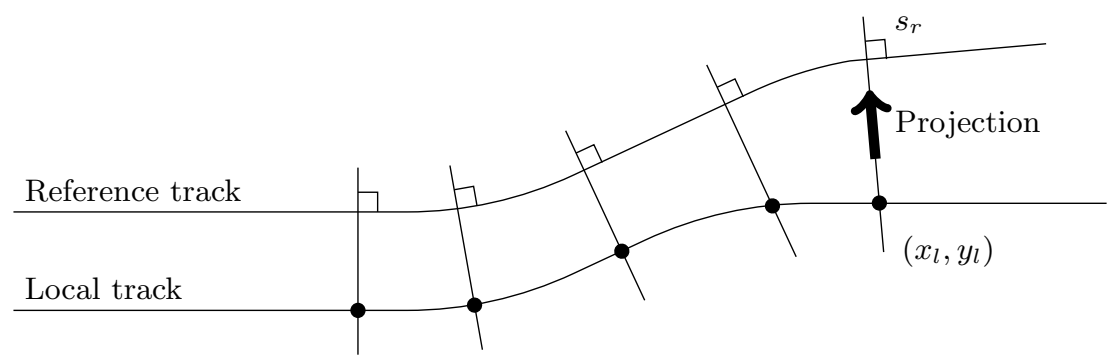

Fig. 2. Linear reference position calculated by projection onto a reference track.

The main contributions of this paper are: (1) We describe and formalize the problem of linear schematic railway drawings in Section 2. (2) We define three mathematical models for schematic plans, and compare their strengths and weaknesses in Section 3. (3) We develop a downloadable tool that can be used by railway engineers to visualize infrastructure, and demonstrate its performance and output on real-world infrastructure models in Section 4. Our tool is meant to be used as a module integrated in the RailCOMPLETE engineering framework; but it can also be used as a standalone tool by researchers and developers working on new techniques for analysis and verification, e.g. on interlockings or capacity and timetabling, who can greatly benefit from low-effort, high-quality visualizations in order to improve communication, usability, and for lowering the barrier for adoption of their tools and techniques. Our tool takes input railML files, which are widely available among railway engineers as it is a standard description format for railway infrastructure. The tool also has options for placing symbols besides a track in the schematics.

\section{Problem definition and formalization}

\subsection{Linear positioning system}

It is a common practice in railway engineering to use a linear reference positioning system, which assigns a scalar value to each point on, or beside, a railway track. The value corresponds approximately to the traveling distance along a railway corridor from a reference point (which is often a known point on the central station of the network). For a single track, the linear reference system may simply be the arc length from the start of the track's center-line curve. Double tracks and side tracks are typically mapped to the linear reference position by geometrically projecting each point onto a reference curve. The projection's target curve may either be a selected reference track (see Fig. 2), or another curve that does not necessarily coincide with a track, such as the geometrical center-line of the corridor. For the rest of this paper, we assume that all locations are already given in such a linear reference system. 


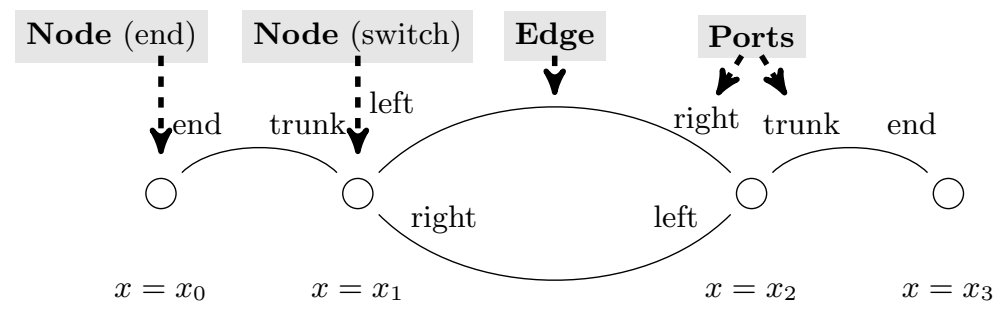

Fig. 3. Graph representation of linearized track plan. Nodes are ordered by an $x$ coordinate, and have a given type which determines which ports it has, e.g., a switch node has trunk, left, and right ports. Edges connect ports on distinct nodes.

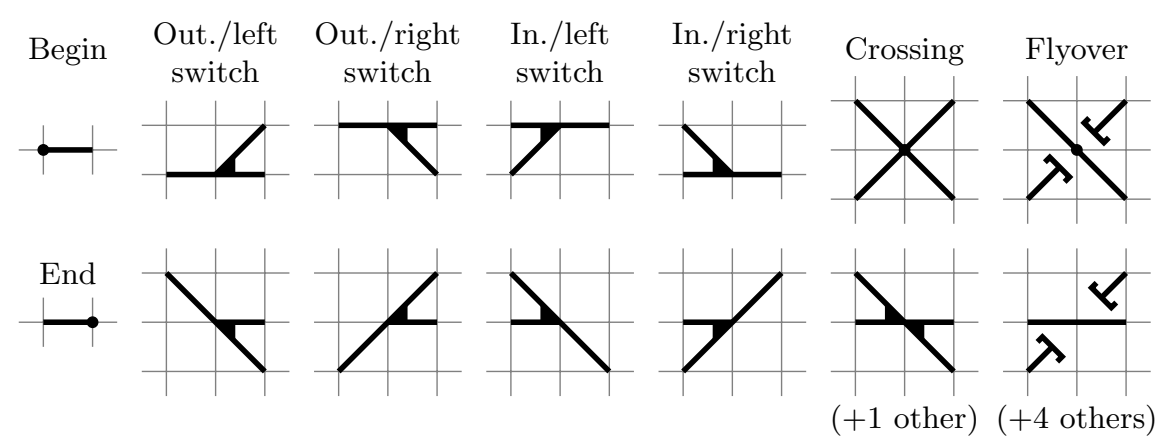

Fig. 4. Node classes and their drawing variants. Begin/end nodes have one variant each. Switches are divided into four classes (each with two variants) based on their orientation (incoming or outgoing) and their course (deviating left or right). Crossings have three variants, and flyovers have six variants (symmetric variants omitted).

\subsection{Track network representation}

Different track segments are connected together at switches in a graph-like network. The mathematical definition of a graph is too abstract for many engineering use cases. Some applications use a double node graph [11], or describe tracks as nodes with two distinct sides [1]. For a schematic plan, we model switches and crossings as graph nodes which have a given set of ports (Fig. 3 presents all our modeling elements). Each end of each edge connects to a specific port on a specific node. Model boundaries and track ends are also represented as nodes with a single port.

Each location where tracks start/end or intersect with other tracks is represented as a node of a given class. The classes used in this paper are ends, switches, crossings, and flyovers (shown in Fig. 4 with all their representative variants). Each class comes with a different set of drawing requirements. For example, a switch is oriented such that its branching edges (left/right) point either up (called an outgoing switch) or down (called an incoming switch), seen in the positive direction of the linear positioning system, and each switch class can be drawn in two different variants, chosen freely, one with the trunk and straight leg directed horizontally and another with the deviating leg directed horizontally. 


\subsection{Linear schematic drawing}

A linear schematic drawing algorithm is a core concept in our formalization.

Definition 1. A linear schematic track drawing algorithm $d:(N, E) \rightarrow L$ assigns a set of line segments $L$ to each edge in the set $E$ of edges connecting the set of nodes $N$, where:

$-N=\left\{n_{i}=\left(c_{i}, s_{i}\right)\right\}$, where $c_{i} \in C$ is a node class, and $s_{i} \in \mathbb{R}$ is a linear position distinct from other nodes' positions.

- $E=\left\{e_{j}=\left(n_{a}, p_{a}, n_{b}, p_{b}\right)\right\}$, where $n_{a}, n_{b} \in N$ are two nodes where $s_{a}<s_{b}$ and $p_{a}, p_{b}$ are distinct, available ports on the referenced nodes.

- $L=\left\{\left(e_{j}, l_{j}\right)\right\}$, where $l_{j}$ is a polyline, representing the drawing of edge $e_{j} \in E$, and defined by a sequence of points in $\mathbb{R}^{2},\left\langle\left(x_{1}^{j}, y_{1}^{j}\right),\left(x_{2}^{j}, y_{2}^{j}\right), \ldots,\left(x_{n}^{j}, y_{n}^{j}\right)\right\rangle$. The polyline consists of the line segments connecting consecutive points in this sequence.

The definition of a track drawing algorithm in itself does not ensure that the output drawing is suitable for reading. To ensure a usable output we establish a set of criteria for drawing quality based on engineering conventions and aesthetic judgements. We divide the criteria into hard constraints, that all drawings must satisfy and that we can base mathematical models on, and soft constraints, which are optimization criteria that can be prioritized differently in different use cases. We base our models on the following hard constraints provided by railway signaling engineers (from Railcomplete AS):

(A) Octilinearity: the lines representing tracks should be either horizontal, or diagonal at $45^{\circ}$. This property contributes to the neat look of a schematic drawing, while also giving a visual clue that the drawing is not fully geometrically accurate. When loops are present in the infrastructure, vertical lines may also be allowed, such as in the balloon loop used on many tram lines.
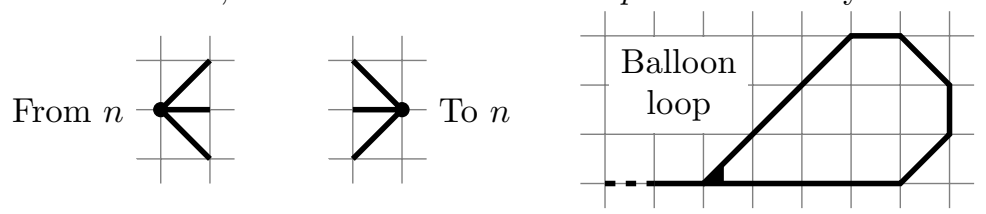

(B) Linear order: the reference mileages of locations on the infrastructure should be ordered left-to-right on the schematic drawing to give a clear sense of sequence, which is useful when navigating the infrastructure and reasoning about train movements.

$$
s_{a}<s_{b} \Rightarrow
$$

(C) Node shapes: switches split the track on the trunk side into a left and a right leg on the branch side. Left and right should be preserved so that the layout can be traced back to the geography. Since one of the legs of the switch is typically straight and the other is curved, it is also desirable to preserve the straight leg's direction relative to the trunk. 


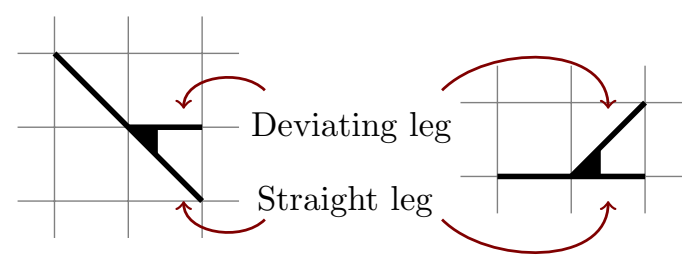

(D) Uniform horizontal spacing: parallel tracks are typically required to be drawn at a specific distance from each other, which we normalize and say that $y$ coordinates take integer values. Note that $x$ coordinates have no such restriction, but consecutive nodes will often be placed at integer-valued distances to fulfill the octilinearity constraint.

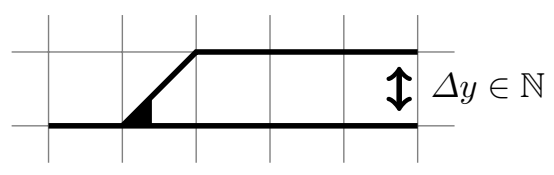

Even with the above constraints fulfilled, there is no guarantee that the drawing output of an algorithm can be deemed of high-quality. For this we use the following soft constraints as optimization criteria:

(i) Width and height of the drawing.

(ii) Diagonal line length, the sum of length of non-horizontal line segments.

(iii) Number of bends, i.e. the number of direction changes on lines.

These criteria have different priorities in different use cases. For example, a signaling schematic might be optimized to have a minimum amount of diagonal lines to neatly show several concurrent train movements and their relative progress, while a dispatch control station schematic might be optimized for width to fit more infrastructure into a computer screen.

Several or all of the criteria can be combined into an optimization objective, either by a scoring function, or more commonly, by simply ordering the objectives and performing lexicographical optimization on each objective in turn. Our tool (detailed in Section 4) provides options for ranking the objectives.

\section{Model definitions and drawing algorithms}

This section describes three different models of linear schematic drawings. First, we present a linear programming formulation where edges can have up to two bends. The resulting optimization problem is efficiently solvable, but has some drawbacks in visual quality. Second, we introduce Boolean choice variables to mitigate the shortcomings of the linear programming formulation, and use instead a SAT solver and lazy solving of difference constraints to optimize the Boolean/numerical model (keeping the maximum of two bends per edge). Finally, we present a different Boolean model with unbounded number of bends per edge, which makes this formulation able to optimize drawing size further 
than the two previous models. However, this comes at the cost of increased running time. Comparison Figures 7, 8, and 11 demonstrate the strengths and weaknesses of each approach, while Table 1 shows their relative performance. All models use a pre-processing step which orders edges vertically, as in Sec. 3.1.

\subsection{Vertical ordering relation on edges}

From the nodes and edges defined as inputs to the linear schematic drawing algorithm, it is possible to derive a vertical ordering relation $<_{E}$ on the set of edges. This relation is a strict partial order relating edges whose linear position intervals intersect, i.e., it relates each pair of edges $e_{a}$ from $n_{a_{l}}$ to $n_{a_{r}}$, and $e_{b}$ from $n_{b_{l}}$ to $n_{b_{r}}$, where:

$$
] s_{a_{l}}, s_{a_{r}}[\cap] s_{b_{l}}, s_{b_{r}}[\neq \emptyset
$$

Such a relation can be established by considering paths starting in each of the branch-side ports of each switch, crossing, and flyover (cf. Fig. 4). For example, an outgoing switch with branch-side edges $e_{a}$ and $e_{b}$ connecting to its right and left ports, respectively, will obviously have $e_{a}<_{E} e_{b}$. Each edge connected to the outgoing edges from the other side of $e_{a}$ and $e_{b}$ will also be ordered vertically, and so on until either of the following termination conditions are fulfilled:

(C1) The two sets of edges meet in another node.

(C2) One of the sides has no more edges to follow.

More precisely, we define $<_{E}$ by the following. Let $G=(N, E)$ be the graph from Definition 1. We first look in the positive direction on the linear reference axis. We define a vertical order relation $<_{E}^{i}$ for each node $n_{i} \in N$. If $n_{i}$ has less than two ports on the side of increasing linear position, $<_{E}^{i}$ is empty. However, if the node has two ports on the side of increasing linear position, let the edges connected to these ports be $e_{l}$, the lower edge, and $e_{h}$, the higher edge. For example, in an outgoing switch node (cf. Fig. 4), these correspond to the right and left ports, respectively.

For any node $n_{j}$ with $s_{i}<s_{j}$, define the directed graph $H_{] i, j}$ containing:

- The subset of nodes from $G$ with positions in the open interval $] s_{i}, s_{j}[$, along with any number of fresh nodes (i.e. the nodes $n_{i}$ and $n_{j}$ are not included).

- The subset of edges from $G$ which have at least one end connected to a node from the open interval $] s_{i}, s_{j}$ [, directed in the direction of increasing linear position. If an edge connects to a node from $G$ which is not included in $H_{] i, j}$, that connection is replaced with a connection to a distinct fresh node.

We are looking for those nodes $n_{j}$ such that, in $H_{] i, j}$, the set of reachable edges when starting from $e_{l}$ are disjoint from the set of reachable edges when starting from $e_{h}$ (termination condition $(\mathrm{C} 1)$ ), see Fig. 5(a). Also, the linear position interval of each edge reachable from $e_{l}$ should have a non-empty intersection with at least one edge reachable from $e_{h}$, and vice versa (termination condition 


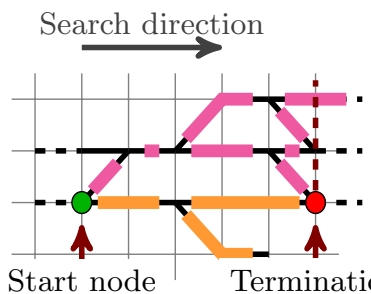

(a)

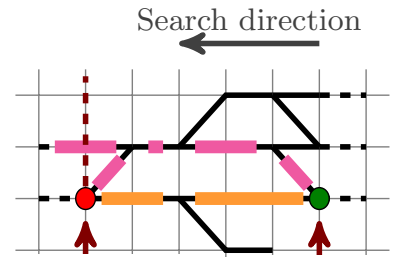

Start node

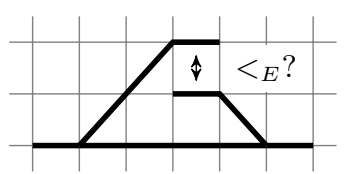

(c) Clothes iron

Fig. 5. A search procedure starting in each node produces a set of tuples for the edge vertical order relation $<_{E}$. Figures (a) and (b) show two different start nodes and search directions, where the lighter, orange edges are all below darker, magenta edges. Figure (c) shows an input on which the procedure cannot decide an ordering.

(C2)). The node $n_{j}$ which has the highest position $s_{j}$ while still fulfilling the above criteria, is called the termination position.

Each edge $e_{x}$ reachable from $e_{l}$ in $H_{] i, j[}$ is below all edges $e_{y}$ reachable from $e_{h}$ in $H_{] i, j}$ [ whenever this pair of edges has intersecting linear position intervals, in which case we have $e_{x}<_{E}^{i} e_{y}$.

For the direction of decreasing linear position we apply the same argument with horizontal directions reversed (see Fig. 5(b)). Finally, the relation $<_{E}$ is defined as the union of the relations from each node,

$$
<_{E}=\bigcup_{n_{i} \in N}<_{E}^{i}
$$

Remark 1. Unconnected graph components must still be explicitly ordered, and the same for some connected topologies such as the clothes iron example in Fig. 5(c). These are usually easy to decide from, e.g., a geographical model, and this situation occurs rarely, in our experience.

\subsection{Level-based linear programming encoding}

We start out by giving a constraint system on linear equations over continuous numerical variables which fulfills the hard requirements from Section 2.3 and can be solved efficiently by linear programming (we used the CBC solver v2.94). Later, the shortcomings of this model will motivate the introduction of Boolean and integer-valued variables and a SAT problem formulation.

For each node $n_{i}$ we use two real variables, $x_{i}$ and $y_{i}$, representing the schematic coordinates of nodes. For each edge $e_{i}$ we use one real variable $l_{i}$ representing the edge's level. This builds in an assumption that each edge is drawn in three parts as explained in Fig. 6. We introduce the following constraints:

1. Node location ordering for successive nodes $n_{i}, n_{j}$ gives $x_{i} \leq x_{j}$, corresponding to the linear order requirement (from Sec. 2.3(B)).

\footnotetext{
${ }^{4}$ Part of the COIN-OR project 2018: https://projects. coin-or.org/Cbc
} 


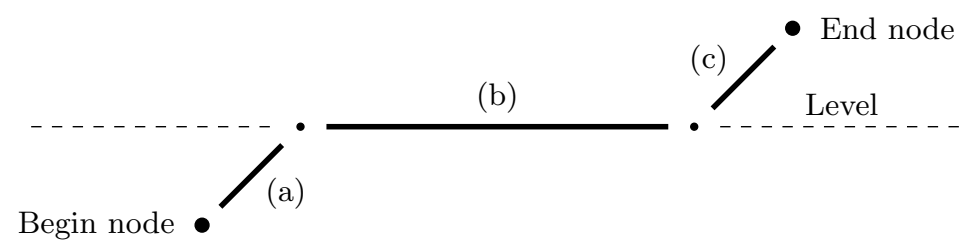

Fig. 6. The edge level model divides the edge into three sections on the horizontal axis: (a) the initial diagonal section from the left-most node to the edge level, (b) the middle horizontal section connecting the two diagonal sections, (c) the final diagonal section reaching the right-most node from the edge level. Any of these may have zero length.

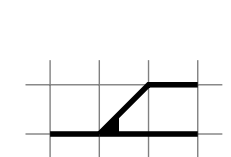

(a) Junction

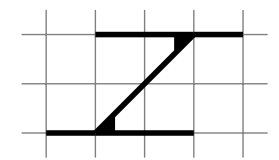

(b) Crossover

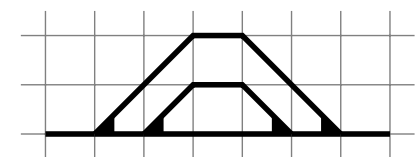

(c) Nested siding loops

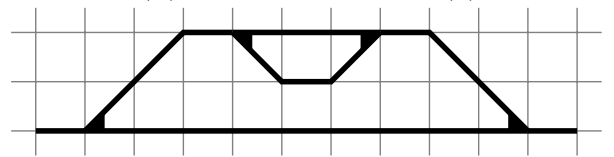

(d) Ladder sidings

Fig. 7. Output examples for the linear programming method. The junction (a) and nested sidings (c) are correctly drawn. The crossover (b) uses 2 units for the diagonal, where 1 would be sufficient, because each edge requires a level distinct from other edges with intersecting linear position intervals. The ladder sidings (d) are unnecessarily wide because node shape variants are not included (compare with Fig. 8(b)).

2. Node location distance for nodes $n_{i}, n_{j}$ connected by an edge $e_{k}$, where $s_{i}<s_{j}$, gives $x_{i}+\left|l_{k}-y_{i}\right|+\left|y_{j}-l_{k}\right|+q_{k} \leq x_{j}$, where $q_{k}$ is 0 if the edge connects an outgoing switch to an incoming switch with the same branching direction, and 1 otherwise. This creates room for a horizontal line segment if needed. The sign of the absolute value terms is determined statically (not part of the linear programming) by the node class and variant. This constraint corresponds to the octilinearity requirement (from Sec. 2.3(A)).

3. Edge level ordering for edges: $e_{i}<_{E} e_{j}$ gives $l_{i}+1 \leq l_{j}$, corresponding to the node shape requirement (from Sec. 2.3(C)).

4. Edge levels are related by switches, i.e.: each switch node $n_{i}$ constrains the trunk-side edge $e_{j}$ and the straight branch-side edge $e_{k}$ to be at the same level as the node $\left(y_{i}=l_{j}=l_{k}\right)$ corresponding to the node shape requirement.

Note that the uniform horizontal spacing constraint (from Sec. 2.3(D)) is implicit in these equations. Now we have the following criteria available for optimization:

- Width of the drawing. Take the node $n_{i}$ with the lowest $s_{i}$, and the node $n_{j}$ with the highest $s_{j}$. Then the width of the drawings is $x_{j}-x_{i}$.

- Height of the drawing. The height of the drawing is not directly expressible in this model, but can be approximated by summing the vertical level 
difference of edges. For pairs of edges $e_{i}, e_{j}$ where $e_{i}<_{E} e_{j}$, the vertical level difference distance is $l_{j}-l_{i}$.

Some output examples from the linear programming solution are shown in Fig. 7. Although efficiently solvable, this linear programming solution has a main drawback in that it is not able to choose between different alternatives for drawing a node. For example, in the so-called ladder configuration shown in Fig. 7(d), much space is wasted on diagonal lines going to the top-most level, when the two topmost switches could have been rotated to produce a simpler drawing. Also, each edge needs to have a $y$ value distinct from other edges with intersecting $x$ intervals, even if it is drawn only with diagonals, such as in Fig. 7(b), which contributes to inefficient use of space. Both these shortcomings will be improved by the level-based Boolean formulation in the next section.

\subsection{Level-based SAT encoding}

We reformulate the problem using variables from the Boolean and bounded integer domains. Since we are dealing with small integers, we can transform the problem into a Boolean satisfiability problem (SAT) by encoding numerical variables into Boolean variables and use incremental SAT solvers which can be efficient for lexicographical optimization on small discrete domains, as ours.

Integers can be encoded into SAT in various ways. Eager encodings represent numbers and constraints directly using a set of Boolean variables and constraints and creates an equisatisfiable SAT instance. Most commonly used is the binary encoding (one Boolean for each bit) and the unary encoding (one Boolean for each distinct number). See [4] for details. Lazy encodings, as used in SMT solvers (see $[3,13]$ for an introduction), can avoid some of the work of transforming and solving a large SAT problem by abstracting the numerical constraints into marker Boolean variables. Only when the SAT solver sets markers to true, another procedure (the theory solver) will go to work on the numerical constraints and report unsatisfiable combinations back to the SAT solver.

Although the SAT problem itself does not directly concern numbers, much less numerical optimization, an incremental interface to a SAT solver allows solving many similar problems consecutively. For a set of constraints $\phi$, we can perform numerical optimization on some number $x$ by solving the sequence of formulas $\phi \wedge\left(x<m_{1}\right), \phi \wedge\left(x<m_{2}\right), \ldots$, where the sequence $m_{i}$ is a linear or binary search over the range of $x$, locating the smallest value that satisfies the constraints. Querying the solver successively with such similar formulas incrementally is much faster than solving the instances separately.

We used the MiniSAT [10] solver v2.2.0 with unary encoding of bounded integers and also lazy representation of unbounded integers with difference constraints, i.e. constraints of the form $x_{i}-x_{j} \leq k$, where $k$ is a constant. Difference constraints are suitable as a first-line refinement in SMT solvers (see e.g. [5]) because they can be efficiently solved.

We keep the assumption from the previous subsection that each edge is assigned to a single level, and extend the problem representation as follows: 


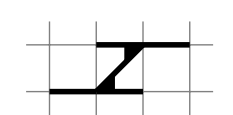

(a) Crossover

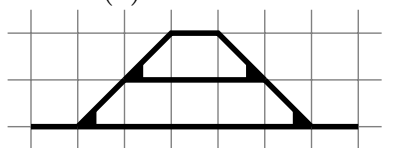

(b) Ladder sidings

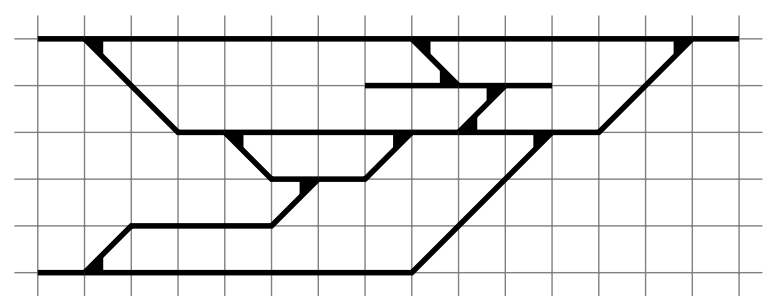

(c) Real-world example: Eidsvoll railML

Fig. 8. Output examples for the level-based SAT method. The crossover (a) requires only a 1 unit diagonal edge (improving Fig. 7(b)). The ladder sidings (b) now use diagonal switch variants to improve width, height, and bends (improving Fig. 7(d)). The Eidsvoll station (c) demonstrates real-world infrastructure imported from railML.

1. Distances between nodes are represented as a saturating unary number of size 2, i.e. $\Delta x \in\{0,1, \geq 2\}$. This allows us to distinguish between short $(\Delta x \leq 1)$ and long $(\Delta x \geq 2)$ edges.

2. For each edge $e_{j}$, we use Booleans $q_{j}^{\text {up }}$ and $q_{j}^{\text {down }}$ to indicate a short edge pointing up/down, respectively, seen in the direction of increasing $x$.

3. Node vertical coordinates $y_{i}$ and edge levels $l_{j}$ are represented by unbounded integers on which we can conditionally impose difference constraints.

4. Variant selection $r_{i} \in R\left(c_{j}\right)$ for each node $i$ indicates the node's variant from the available shapes $R\left(c_{j}\right)$ of the node class $c_{j} \in C$ listed in Fig. 4.

5. Edge direction values, $d_{i}^{\text {begin }}, d_{i}^{\text {end }} \in\{\mathrm{Up}$, Straight, Down $\}$, for the beginning and end of each edge $e_{i}$, are based on node variant values.

We need the following constraints:

- Each edge must be at least 1 unit long on the $\mathrm{x}$ axis.

- Edge ordering constraints for $e_{a}<_{E} e_{b}$ :

$$
l_{a} \leq l_{b}, \quad\left(\neg q_{a}^{\text {up }} \wedge \neg q_{b}^{\text {down }}\right) \Rightarrow l_{a}+1 \leq l_{b}
$$

If an edge is a short edge (such as a crossover between two adjacent tracks) it does not require its own level, and we use instead the same level as the one of its end nodes which has the highest value. This allows to produce a better crossover drawing, as in Fig. 8(a) instead of Fig. 7(b).

- An edge $i$ is short ( $q^{\text {up }}$ or $\left.q^{\text {down }}\right)$ if both ends have the same direction and the vertical distance between nodes is one:

$$
\begin{gathered}
q_{i}^{\text {up }} \Rightarrow\left(d_{i}^{\text {begin }}=\mathrm{Up}\right) \wedge\left(d_{i}^{\text {end }}=\mathrm{Up}\right) \wedge\left(y_{a}+1=y_{b}\right) \\
q_{i}^{\text {down }} \Rightarrow\left(d_{i}^{\text {begin }}=\text { Down }\right) \wedge\left(d_{i}^{\text {end }}=\text { Down }\right) \wedge\left(y_{a}-1=y_{b}\right)
\end{gathered}
$$

- Direction on edge $i$ decides vertical level constraints:

$$
\begin{gathered}
\left(d_{i}^{\text {begin }}=\text { Straight }\right) \Rightarrow\left(y_{a}=l_{i}\right), \quad\left(d_{i}^{\text {begin }}=\mathrm{Up}\right) \Rightarrow y_{a}+1 \leq l_{i}, \\
\left(d_{i}^{\text {begin }}=\text { Down }\right) \Rightarrow\left(\left(q_{i}^{\text {up }} \Rightarrow\left(y_{a} \geq l_{i}\right)\right) \wedge\left(\neg q_{i}^{\text {up }} \Rightarrow\left(y_{a} \geq l_{i}+1\right)\right)\right)
\end{gathered}
$$

And correspondingly for $d^{\text {end }}$. 
- The sum of $\Delta x$ values over the edge must match the shape of the edge:

$$
\begin{gathered}
\left(q^{\text {up }} \vee q^{\text {down }}\right) \Rightarrow \Sigma_{j \in(a, b)} \Delta x_{j} \leq 1 \\
\left(\neg q^{\text {up }} \wedge \neg q^{\text {down }} \wedge\left(d^{\text {begin }} \neq \text { Straight } \vee d^{\text {end }} \neq \text { Straight }\right)\right) \Rightarrow \Sigma_{j \in(a, b)} \Delta x_{j} \geq 2
\end{gathered}
$$

Since the shape of an edge is now explicit through $d^{\text {begin }}$ and $d^{\text {end }}$, we can optimize for the number of bends to produce Fig. 8(b) instead of Fig. 7(d).

The level-based representations do not represent the shapes of edges explicitly at each coordinate, and thus cannot insert bends at arbitrary locations, something which is needed to pack drawings together more tightly. A straightforward grid-based SAT encoding could associate each point on a grid with a choice of any node, and each cell with a choice of edge shape. With this encoding, however, drawings with only about 30 nodes take hours to optimize. We do not describe this method in more detail here, but we have implemented it and tested its performance compared to the other methods, as shown in Table 1.

\subsection{Cross-section SAT encoding}

Instead of directly representing a grid, we define a vertical cross-section $c_{k}$ of the drawing, represented by a unary-encoded integer $y_{e_{i}}^{k}$ capturing the height of each edge $e_{i}$ at some horizontal location in the drawing. This naturally allows us to use the edge vertical order $<_{E}$ as constraints on unary numbers $y_{e_{i}}^{k}<_{E} y_{e_{j}}^{k}$. Each pair of successive nodes is transformed into a sequence of such cross-sections, and we associate a direction $d_{e_{i}}^{k} \in\{\mathrm{Up}$, Straight, Down $\}$ with each edge $e_{i}$ at each cross-section $c_{k}$, giving the shape of the edge to the left (lower $x$ value) of the cross-section. Cross-sections can be enabled or disabled (represented by $b_{k}$ ) to optimize the width of the drawing. Finally, the ahead Boolean $a_{e_{i}}^{k}$ for each edge at each cross-section marks whether the shape of the edge has already been constrained for the next cross-section to the right (higher $x$ value), which allows nodes to impose edge shape constraints in both $x$-axis directions.

With this representation, we can impose constraints as follows:

1. Edge vertical order:

$$
\left(e_{i}<_{E} e_{j}\right) \Rightarrow \bigwedge_{c_{k}} y_{e_{i}}^{k} \leq y_{e_{j}}^{k}
$$

2. A begin node at cross-section $c_{k}$ constrains the edge shape to the right, and makes the $y$ value unequal to the $y$ value of other edges $e_{j} \in c_{k}$.

$$
a_{e_{i}}^{k} \wedge d_{e_{i}}^{k}=\text { Straight }, \quad \bigwedge_{e_{j} \in c_{k}} y_{e_{i}}^{k} \neq y_{e_{j}}^{k},
$$

and similar for end nodes, in the opposite direction:

$$
\neg a_{e_{i}}^{k} \wedge d_{e_{i}}^{k}=\text { Straight, } \quad \bigwedge_{e_{j} \in c_{k}} y_{e_{i}}^{k} \neq y_{e_{j}}^{k} .
$$


3. A switch node at cross-section $c_{k}$ constrains the edge shape in both directions by constraining the incoming edges $e_{i}$ according to the node class variant. For example, for an outgoing left switch we have one incoming edge $e_{i 1}$ :

$$
\neg a_{e_{i} 1}^{k} \wedge d_{e_{i} 1}^{k} \neq \mathrm{Up}
$$

The incoming edges $e_{i}$ are replaced by the outgoing edges $e_{j}$ in the crosssection representation. For example, for an outgoing left switch (see Fig. 9) we have two outgoing edges $e_{j 1}, e_{j 2}$ as the left and right ports, respectively:

$$
a_{e_{j 1}}^{k} \wedge a_{e_{j 2}}^{k}
$$

and we have two choices of shape:

$$
\begin{gathered}
\left(d_{e_{i}}^{k}=\text { Straight }\right) \Rightarrow\left(y_{e_{i}}^{k}=y_{e_{j}}^{k} \wedge d_{e_{j 2}}^{k}=\text { Straight } \wedge d_{e_{j 1}}^{k}=\text { Left }\right) \\
\left(d_{e_{i}}^{k}=\text { Down }\right) \Rightarrow\left(y_{e_{i}}^{k}=y_{e_{j 1}}^{k} \wedge d_{e_{j 2}}^{k}=\text { Down } \wedge d_{e_{j 1}}^{k}=\text { Straight }\right)
\end{gathered}
$$

Constraints are similar for other node classes.

4. Disabled cross-sections propagate all their values:

$$
\neg b_{k} \Rightarrow \bigwedge_{e_{i} \in c_{k}}\left\{y_{e_{i}}^{k}=y_{e_{i}}^{k+1} \wedge a_{e_{i}}^{k}=a_{e_{i}}^{k+1} \wedge d_{e_{i}}^{k}=d_{e_{i}}^{k+1}\right\}
$$

5. Enabled cross-sections require consistency between edge shapes and $y$ values:

$$
b_{k} \Rightarrow \bigwedge_{e_{i} \in c_{k}}\left\{\left(\neg a_{e_{i}}^{k} \wedge d_{e_{i}}^{k+1}=\mathrm{Up}\right) \Rightarrow y_{e_{i}}^{k}+1=y_{e_{i}}^{k+1}\right\}
$$

And correspondingly for Straight and Down directions.

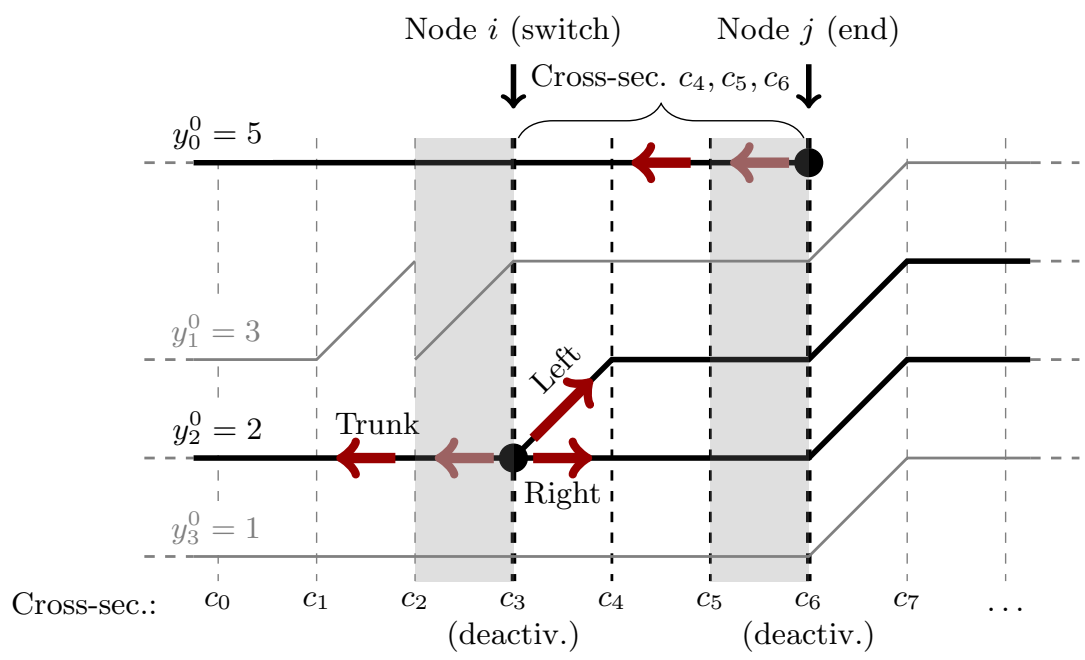

Fig. 9. Cross-section SAT representation. Dashed vertical lines show cross-sections $c_{i}$. Edges have a $y$ value and a direction to the left of each cross-sec. Thick red arrows are constraints imposed by node type. Gray columns correspond to deactivated crosssections, where shape constraints are propagated to the next or previous column. 


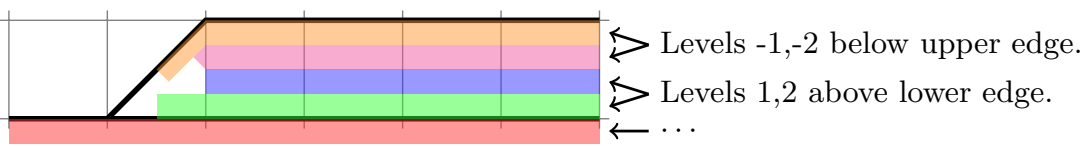

Fig. 10. Label placement can be done by restricting symbols to fit into a set of levels above and below each line, which reduces the constraints to linear ordering.

6. Enabled cross-sections realize rightward-constrained ahead values $a$ :

$$
b_{k} \Rightarrow \bigwedge_{e_{i} \in c_{k}}\left\{\left(a_{e_{i}}^{k} \Rightarrow y_{e_{i}}^{k}=y_{e_{i}}^{k+1}\right) \wedge\left(a_{e_{i}}^{k} \Rightarrow d_{e_{i}}^{k}=d_{e_{i}}^{k+1}\right) \wedge \neg a_{e_{i}}^{k+1}\right\}
$$

With this formulation we can choose freely between prioritizing width, height, or bends, and the resulting plans have lower total width than for the level-based methods, since the grid-based method has the added freedom of inserting bends at any location along an edge. See Fig. 11 for a comparison.

\subsection{Symbols and labels}

A railway engineering schematic often features a large amount of different symbols and labels (see the example in Fig. 1). In some cases, the symbols and labels can be placed onto a well laid-out track plan without needing to change the track plan, but there are common cases where the track layout must be drawn in a way that accounts for the amounts and sizes of symbols and labels. Our tool has options for placing symbols into two rows above and below each track, which is suitable for signaling drawings.

Label placement in general is known as a hard problem in graph visualization. We use a simplified approach suitable for thin rectangular symbols (e.g. as in Fig. 1), and assign each symbol to a level above or below the track (see Fig. 10). Difference constraints on $x$ values ensure that symbols are ordered and not overlapping. When constraints are satisfied, we use linear programming to minimize the deviation from proportional distance between nodes, so that symbols are close to each other on the drawing if they are physically close.

\section{Tool usage}

A command-line tool that can generate the drawings as described in this paper is available online ${ }^{5}$. The tool can import railML files as track network input and track-side object symbols, or use a custom format for directly specifying topology. The tool offers two choices of built-in symbol appearances: "simple" for generic lamp-like signals and detector, and "ERTMS" for ERTMS-style marker boards and detectors (see the bottom part of Fig. 11). Extracting other object types from railML and producing other symbol styles can be done by postprocessing JSON output, or by extending the tool using the Lua scripting language. The tool can produce output in JSON format (for custom visualization

\footnotetext{
${ }^{5}$ https://github.com/luteberget/railplot
} 
Model: Eidsvoll, imported from BaneNOR railML [19]

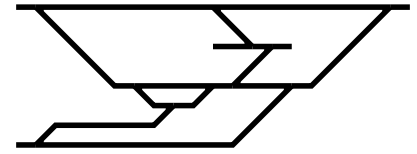

Levels/Lin.Prog

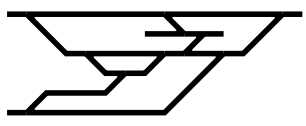

Levels/SAT

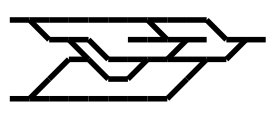

Cross-sec./SAT, opt. width/height

Model: Asker, imported from BaneNOR railML [19]

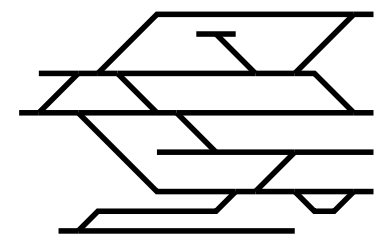

Levels/Lin.Prog

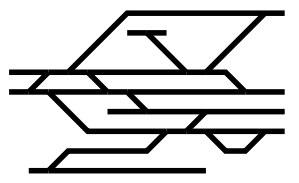

Levels/SAT

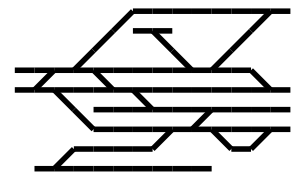

Cross-sec./SAT, opt. height/bends

Model: Arna, imported from RailCOMPLETE CAD project

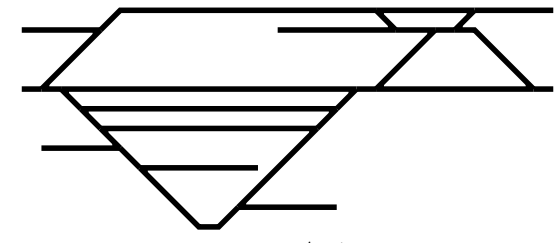

Levels/SAT

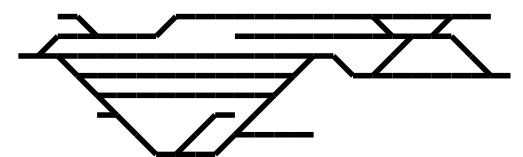

Cross-sec./SAT, opt. height/bends

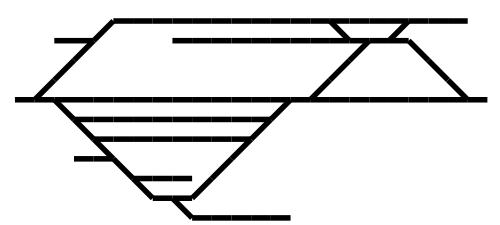

Cross-sec./SAT, opt. bends/width

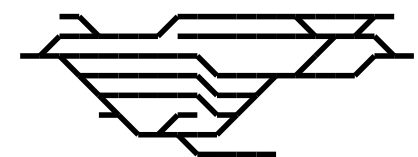

Cross-sec./SAT, opt. height/width Model: Weert, remodeled from figures in [6]

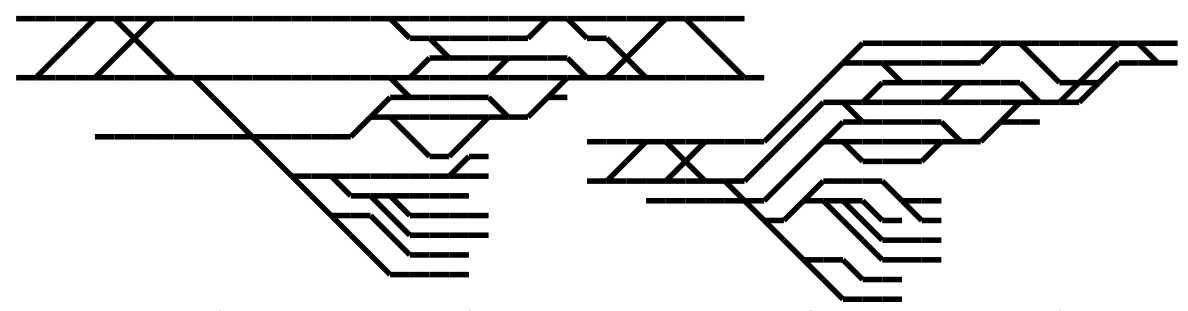

Cross-sec./SAT, opt. height/bends Cross-sec./STT, opt. height/width
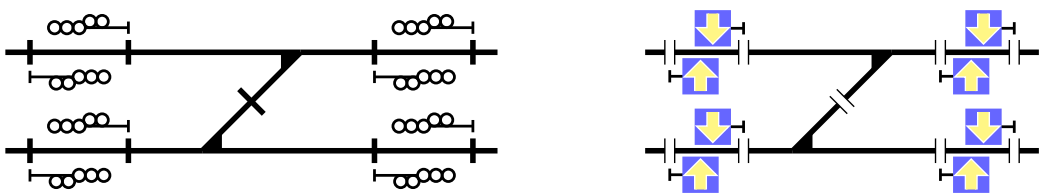

Symbol placement and style affects track layout

Fig. 11. Comparison of three optimization models on various infrastructure models: Levels/Lin.Prog. (see Sec. 3.2), Levels/SAT (see Sec. 3.3), Cross-sec./SAT (see Sec. 3.4). Symbols and labels placed on the drawing may also affect layout (see Sec. 3.5). 


\begin{tabular}{ccc|cccc|cccc} 
Model & \multirow{2}{*}{ Src. Size } & \multicolumn{3}{|c}{ Direct/SAT } & \multicolumn{3}{c}{ Levels/SAT } & \multicolumn{4}{c}{ Cross-sec./SAT } \\
& & & hwb & size (v/c) & bhw & size (v/c) & hwb & hbw & bhw & size (v/c) \\
\hline Eidsvoll & {$[19]$} & 35 & 60.7 & $57 \mathrm{k} / 153 \mathrm{k}$ & 0.02 & $2.3 \mathrm{k} / 0.7 \mathrm{k}$ & 0.05 & 0.06 & 0.33 & $4.0 \mathrm{k} / 28 \mathrm{k}$ \\
Arna & $\mathrm{RC}$ & 57 & 294 & $167 \mathrm{k} / 493 \mathrm{k}$ & 0.03 & $4.9 \mathrm{k} / 1.3 \mathrm{k}$ & 0.26 & 0.65 & 1.06 & $11 \mathrm{k} / 100 \mathrm{k}$ \\
Asker & {$[19]$} & 64 & $\mathrm{~T} / \mathrm{O}$ & $104 \mathrm{k} / 295 \mathrm{k}$ & 0.04 & $5.6 \mathrm{k} / 2.0 \mathrm{k}$ & 0.61 & 1.02 & 0.87 & $14 \mathrm{k} / 124 \mathrm{k}$ \\
Weert & {$[6]$} & 102 & $\mathrm{~T} / \mathrm{O}$ & $304 \mathrm{k} / 969 \mathrm{k}$ & 0.18 & $11 \mathrm{k} / 4.0 \mathrm{k}$ & 0.72 & 19.3 & 21.4 & $29 \mathrm{k} / 327 \mathrm{k}$ \\
\hline $5 \times 10$ & $\mathrm{~T}$ & 228 & $\mathrm{~T} / \mathrm{O}$ & $2.8 \mathrm{M} / 13 \mathrm{M}$ & 0.58 & $35 \mathrm{k} / 2.7 \mathrm{k}$ & 5.83 & 7.48 & 8.08 & $46 \mathrm{k} / 364 \mathrm{k}$ \\
$5 \mathrm{x} 0$ & $\mathrm{~T}$ & 478 & $\mathrm{~T} / \mathrm{O}$ & $2.8 \mathrm{M} / 12 \mathrm{M}$ & 3.37 & $97 \mathrm{k} / 7.7 \mathrm{k}$ & 279 & 299 & $\mathrm{~T} / \mathrm{O}$ & $265 \mathrm{k} / 4.2 \mathrm{M}$ \\
$10 \mathrm{x} 5$ & $\mathrm{~T}$ & 203 & $\mathrm{~T} / \mathrm{O}$ & $3.0 \mathrm{M} / 14 \mathrm{M}$ & 0.40 & $28 \mathrm{k} / 2.0 \mathrm{k}$ & 0.52 & 0.59 & 1.08 & $20 \mathrm{k} / 83 \mathrm{k}$ \\
$20 \mathrm{x} 5$ & $\mathrm{~T}$ & 403 & $\mathrm{~T} / \mathrm{O}$ & $3.0 \mathrm{M} / 14 \mathrm{M}$ & 1.73 & $70 \mathrm{k} / 4.0 \mathrm{k}$ & 1.95 & 2.50 & 3.36 & $44 \mathrm{k} / 165 \mathrm{k}$ \\
$10 \mathrm{x} 10$ & $\mathrm{~T}$ & 453 & $\mathrm{~T} / \mathrm{O}$ & $2.6 \mathrm{M} / 12 \mathrm{M}$ & 2.74 & $86 \mathrm{k} / 5.5 \mathrm{k}$ & 21.9 & 22.4 & 40.7 & $96 \mathrm{k} / 727 \mathrm{k}$ \\
$15 \mathrm{x} 15$ & $\mathrm{~T}$ & 1053 & $\mathrm{~T} / \mathrm{O}$ & $2.3 \mathrm{M} / 10 \mathrm{M}$ & 22.7 & $255 \mathrm{k} / 15 \mathrm{k}$ & $\mathrm{T} / \mathrm{O}$ & $\mathrm{T} / \mathrm{O}$ & $\mathrm{T} / \mathrm{O}$ & $\mathrm{N} / \mathrm{A}$
\end{tabular}

Table 1. Running times in seconds on a mid-range workstation. Time-outs (T/O) indicate exceeding $300 \mathrm{~s}$. Model sizes are given as the sum of the number of nodes and edges. Models were obtained from BaneNOR [19], a RailCOMPLETE CAD project (RC), and adapted from [6]. Scaling test models (T) named $n \times m$ consist of $n$ serially connected stations, each spreading out to $m$ parallel tracks. Optimization criteria are height (h), width (w) and bends (b). The size columns show the number of SAT variables and clauses $(\mathrm{v} / \mathrm{c})$.

or post-processing), SVG (for use in web pages and web applications), or TikZ (for use in LaTeX documents).

We have implemented and compared the performance of the above SATbased methods, summarized in Table 1 (the linear programming formulation is omitted for space, since it has lower quality output). The Direct/SAT encoding has too poor performance to be of practical value. The Levels/SAT encoding is the fastest, and produces good output when optimizing for bends first. Crosssec./SAT is slower, but is more capable for optimizing for height and width.

\section{Conclusions and Future Work}

We have demonstrated the feasibility of using an incremental SAT solver to automatically produce and optimize schematic railway drawings using several different optimization criteria. However, the choice of encoding makes a significant difference in the size of models that can be handled in a reasonable amount of time, cf. Table 1. The direct representation using an explicit grid fails to handle instances of relevant scale. Only after reformulating the problem in a more structured solution space, where the order of symbols is hard-coded into the problem, rather than added as a constraint after the fact, we were able to solve industrial-size instances in reasonable time for interactive use (i.e., under 1s). A remaining interesting problem is the study of the inherent computational complexity of the linear schematic drawing problem.

Our goal is that professionals should be able to rely on high-quality automatic schematics, which requires further tailoring of symbol and text placement to specific use cases, and integration with GUI tools. 


\section{References}

1. IRS 30100: RailTopoModel - railway infrastructure topological model. The International Union of Railways (UIC), 2016.

2. S. Avelar. Schematic Maps on Demand - Design, Modeling and Visualization. PhD thesis, ETH Zürich, 2002.

3. C. Barrett and C. Tinelli. Satisfiability modulo theories. In Handbook of Model Checking, pages 305-343. Springer, 2018.

4. M. Björk. Successful SAT encoding techniques. JSAT, 7(4):189-201, 2011.

5. M. Bozzano, R. Bruttomesso, A. Cimatti, T. A. Junttila, P. van Rossum, S. Schulz, and R. Sebastiani. An incremental and layered procedure for the satisfiability of linear arithmetic logic. In Tools and Algorithms for the Construction and Analysis of Systems (TACAS), volume 3440 of LNCS, pages 317-333. Springer, 2005.

6. A. Brands. Automatic generation of schematic diagrams of the Dutch railway network. M.Sc. thesis, Radboud University, 2016.

7. S. Cabello, M. de Berg, and M. J. van Kreveld. Schematization of networks. Comput. Geom., 30(3):223-228, 2005.

8. G. Di Battista, P. Eades, R. Tamassia, and I. G. Tollis. Algorithms for drawing graphs: an annotated bibliography. Comput. Geom., 4:235-282, 1994.

9. G. Di Battista, P. Eades, R. Tamassia, and I. G. Tollis. Graph Drawing: Algorithms for the Visualization of Graphs. Prentice-Hall, 1999.

10. N. Eén and N. Sörensson. An extensible SAT-solver. In SAT conference 2003, volume 2919 of LNCS, pages 502-518. Springer, 2003.

11. D. Hürlimann. Objektorientierte Modellierung von Infrastrukturelementen und Betriebsvorgängen im Eisenbahnwesen. PhD thesis, ETH Zurich, 2002.

12. B. Luteberget, K. Claessen, and C. Johansen. Design-time railway capacity verification using SAT modulo discrete event simulation. In FMCAD. IEEE, 2018.

13. R. Nieuwenhuis, A. Oliveras, and C. Tinelli. Solving SAT and SAT modulo theories: From an abstract Davis-Putnam-Logemann-Loveland procedure to DPLL(T). $J$. ACM, 53(6):937-977, 2006.

14. M. Nöllenburg and A. Wolff. Drawing and labeling high-quality metro maps by mixed-integer programming. IEEE Tr.Vis.Comput.Graph., 17(5):626-641, 2011.

15. M. Nöllenburg. Automated drawing of metro maps. Technical Report 25, Universität Karlsruhe, Karlsruhe, 2005.

16. O. Oke and S. Siddiqui. Efficient automated schematic map drawing using multiobjective mixed integer programming. Computers \& OR, 61:1-17, 2015.

17. M. M. Ozdal. Routing Algorithms for High-Performance VLSI Packaging. PhD thesis, 2005.

18. A. Papakostas and I. G. Tollis. Algorithms for area-efficient orthogonal drawings. Comput. Geom., 9(1-2):83-110, 1998.

19. Bane NOR: Model of the Norwegian rail network. http://www.banenor.no/en/ startpage1/Market1/Model-of-the-national-rail-network/, 2016.

20. S. Seyedi-Shandiz. Schematic representation of the geographical railway network used by the Swedish transport administration. M.Sc. thesis, Lund University, 2014.

21. R. Tamassia. On embedding a graph in the grid with the minimum number of bends. SIAM J. Comput., 16(3):421-444, 1987.

22. T. C. van Dijk, F. Lipp, P. Markfelder, and A. Wolff. Computing Storyline Visualizations with Few Block Crossings. In GD, pages 365-378. Springer, 2018.

23. A. Wolff. Drawing subway maps: A survey. Inf. Forsc. Entwick., 22(1):23-44, 2007. 